\title{
Fuzzy Regression Control Chart Based on $\alpha$-cut Approximation
}

\author{
Sevil Şentürk \\ Statistics Department, Anadolu University, Yunusemre Campus 26470 \\ Eskişehir, Turkey \\ E-mail: $\underline{\text { sdeligoz@anadolu.edu.tr }}$ \\ www.anadolu.edu.tr \\ Received: 16-03-2009 \\ Accepted: 08-02-2010
}

\begin{abstract}
The fuzzy regression control chart is a functional technique to evaluate the process in which the average has a trend and the data represents a linguistic or approximate value. In this study, the theoretical structure of the “ $\alpha$-level fuzzy midrange for $\alpha$-cut fuzzy $\widetilde{\bar{X}}$-regression control chart" is proposed for triangular (TFN) and trapezoidal (TrFN) membership functions. In addition, the real world application is evaluated with fuzzy $\widetilde{\bar{X}}$ regression control charts and fuzzy $\widetilde{R}$ control charts for the triangular membership function.
\end{abstract}

Keywords: fuzzy sets, fuzzy numbers, control charts, regression control chart, tool wearing.

\section{Introduction}

Statistical process control (SPC) is a method that uses several types of control charts to measure and monitor product quality. SPC is widely employed throughout industry and is a proven technique for improving quality and productivity. Control charts were proposed by W.A. Shewhart in the 1920 s to evaluate the quality characteristics in products.

Shewhart's control charts evaluate the data representing quality characteristics of the product. If the quality characteristic is represented in a qualitative form, attribute control charts are used to evaluate the process, such as $p, n p, c$ and $u$ control charts. If the quality characteristic is measurable on numerical scales, then control charts for variables, like an $\bar{X}$ chart for the process average and $R$ and $S$ charts for process variability, can be used ${ }^{1}$.

A control chart contains three parts: a centerline that represents the average of the quality characteristics and two other lines, called the upper control limit (UCL) and lower control limit (LCL). The conventional control charts for the quantitative form such as $\bar{X}-R$ consider the average performance of related quality characteristics, and the upper and lower control limits are parallel to the center line. However, in some cases, the related quality characteristics have an increasing or 
decreasing trend because of the tools used in the manufacturing process. For example, an insert is used for the cutting process, making the inner diameter smaller and the external diameter larger due to the blunting and the life of the tool. Because replacing tools may be expensive and time consuming, a certain amount of drift can be tolerated in the process until it reaches the upper and lower specifications. When these dimensions are close to the specifications, the tool should be exchanged for a new one. This is called the tool wearing problem.

Monitoring the tool wear condition is crucial to improve the quality of the manufacturing systems employing drilling and cutting processes. In this case the regression control chart is implemented to examine the tool wearing problem. When tool wear occurs, the process variability at any one point in time is considerably less than the allowable variability over the entire life of the tool. Furthermore, as the tool wears out, there will generally be an upward drift or trend in the mean caused by the worn tool producing larger dimension. The control chart for the tool wearing problem is discussed in more detail in Duncan ${ }^{3}$, Manuele ${ }^{4}$, Mandel $^{5}$ and Quesenberry ${ }^{6}$.

In the regression control chart, the center line is the regression line, $Y=\beta_{0}+\beta_{1} t$, instead of the average of the dimension. Although the center line and upper and lower control limits are parallel to the $X$-axis in the conventional control charts, they are not parallel to the $X$-axis in the regression control chart; see respectively Figure 1 and Figure 2. The process characteristic is called the dependent variable due to the center line, which is represented by the regression equation as seen in the regression control chart. The center line and upper and lower control limits of the regression control chart are represented as a trend equation, as given in Eq. 911.

In tool wear problems, some uncertainty or vagueness arises from the process or measurement system, which includes operators and gauges or environmental conditionals. These uncertainties are based on the process and measurement system, which can lead to some difficulties in obtaining crisp values from the process. In this situation, fuzzy control charts are useful tools for evaluating fuzzy data. Fuzzy set theory can be adopted for the fuzzy control charts. In this condition, fuzzy set theory specifically addresses the development of concepts and techniques for dealing with sources of uncertainty or imprecision.

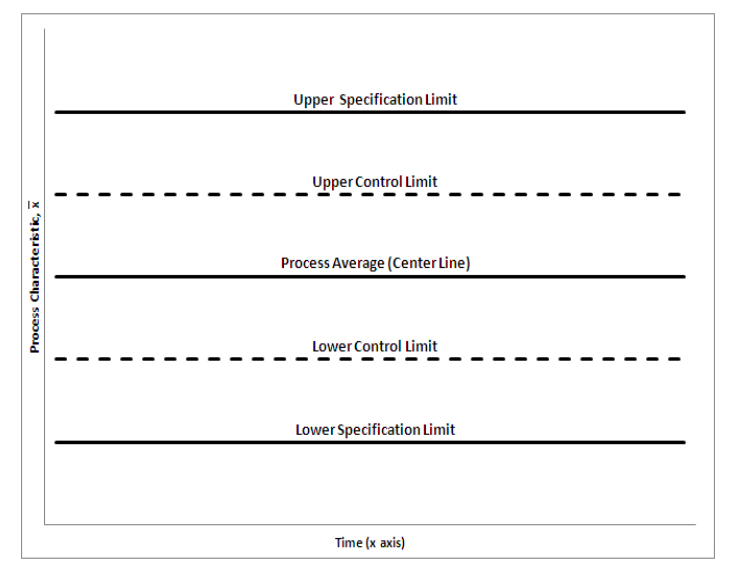

Fig1. Schematic representation of conventional control chart.

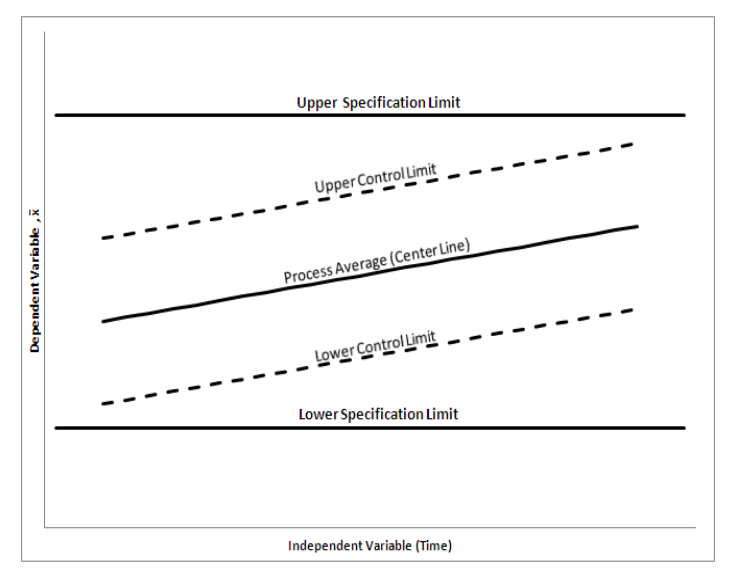

Fig 2. Schematic representation of regression control chart.

Fuzzy set theory was introduced first by Zadeh $^{7}$. Fuzziness in data can refer to various types of vagueness and uncertainty but particularly to the vagueness related to human linguistics and thinking or the lack of information or measurement systems. Many studies have been done to combine several statistical methods and fuzzy set theories, called fuzzy statistics, such as design of experiments, time series analysis, regression analysis, probability theory, conjoint analysis, correspondence analysis and control charts.

Similar to other fuzzy control charts ${ }^{8,9,14,16,17}$, the steps of a fuzzy regression control chart are proposed and an application is presented in this study. When the sample mean has a trend or is too close to the control limits or the measurement system is not very sensitive, the evaluation of processes with a fuzzy regression control chart is more appropriate than the conventional regression 
chart. The main advantages of using fuzzy set theory with a regression control chart are providing flexibility for control limits and reducing false alarms.

The concept of the fuzzy process control chart is well documented in the available literature. The fuzzy control chart for linguistic data was presented by Wang and $\operatorname{Raz}^{8}$. They attempted to extend the use of control charts for linguistic variables by presenting several ways of determining the center line and control limits. Raz and Wang ${ }^{9}$ proposed two approaches for determining the center line and control limits: a probabilistic approach and a membership approach. In the probabilistic approach, the observations are converted from linguistic terms to their crisp values, and the control limits are calculated as a control limit for variables. In the membership approach, the fuzzy subsets corresponding to the observations in a sample are combined into a single fuzzy subset that corresponds to the sample average, according to the rules of fuzzy arithmetic. They conclude with simulated data for control charts based on linguistic data that are significantly more sensitive to process shifts than conventional $p$ charts. Kanagawa et al. ${ }^{10}$ modified the control chart given by Ref. 8 by considering not only the process average but also the process variability. They developed control charts for linguistic variables based on probability density functions that exist behind the linguistic data to control the process variability as well as the process average. This approach is different than the probability density function employed in Ref. 8 . Rowlands and Wang ${ }^{11}$ proposed a method that explores the integration of fuzzy logic and control charts to create and design a fuzzy-SPC evaluation and control method based on the application of fuzzy logic to the SPC zone rules. El-Shal and Morris $^{12}$ presented a control chart by integrating fuzzy logic and SPC zone rules with the aim of reducing the generation of false alarms and also improving the detection and detection-speed of real faults. Hsu and Chen ${ }^{13}$ gave the concept of a fuzzy set by modifying Nelson's rules. The unnatural patterns of symptoms on $\bar{X}$ control charts are indicated by the modified Nelson's rules. In addition, they presented a cause-symptom relation in a fuzzy relation matrix form. Gülbay et al. ${ }^{14}$ proposed an $\alpha$-cut control chart to regulate the tightness of the inspection to attributes with triangular fuzzy numbers to reflect the vagueness of the data. Cheng ${ }^{15}$ proposed an alternative approach to deal with experts' subjective judgments. Based on the rating scores assigned by the individual inspectors to the inspected items, fuzzy numbers are constructed to represent the vague outcomes of the process. Fuzzy control charts are then constructed directly from these fuzzy numbers, thereby retaining the fuzziness of the original vague observations. The out-of-control conditions are then formulated using possibility theory. Gülbay and Kahraman $^{16,17}$ proposed an alternative approach to the fuzzy control chart: the direct fuzzy approach. They used a direct fuzzy approach to fuzzy control chart for attributes under vague data using the probabilities of fuzzy events without any defuzzification. Faraz and Moghadam ${ }^{18}$ introduced a fuzzy chart for controlling the process mean. They designed a fuzzy chart that has a warning line besides the upper control limit. Their method is a more practical method of controlling the process average of a fuzzy control chart, which provides a neural view of the inspectors and offers different strategic options for the company to choose. They detect the desire shifts more quickly, and it is more sensitive to small shifts without any complexity augmentation to the chart. Hsieh et al. ${ }^{19}$ presented a control chart that applies fuzzy theory and engineering experience to monitor wafer defects while considering defect clustering. Their proposed control chart is simpler and more rational than revised c-charts. Zarandi et al. ${ }^{20}$ proposed fuzzified sensitivity criteria and fuzzy adaptive sampling rules with a hybrid adaptive sampling run rules method based on the concepts of fuzzy sets for $\mathrm{X}$ control charts. The two main characteristics of the proposed method are (1) dynamically varying the design parameters, namely sample size and sampling "interval and (2) recognizing nonrandom patterns of the control chart simultaneously. The proposed method is developed based on two strategies: combining adaptive sampling and run rules procedures and using fuzzy adaptive sampling and fuzzy run rules instead of crisp adaptive sampling and crisp run rules. Engin et al. ${ }^{21}$ developed a fuzzy model for attribute control charts (ACC) in multistage processes. The formulation of this model was calculated based on an acceptance sampling approach. Two main parameters were determined for every stage by using genetic algorithms (GAs). The proposed approach was applied in an engine valve manufacturing firm, and the model was solved by Gas. The theoretical structure of fuzzy individual and moving range control charts with $\alpha$-cuts based on an $\alpha$-level fuzzy median transformation technique were developed 
by Erginel $^{22}$ to consider the fuzziness that comes from the measurement system, including operator, gauge, and environmental conditions, which may produce "uncertain" or "vague" data. Adel et al. ${ }^{23}$ proposed a new hybrid approach to estimate change-points in the mean of normal processes for both fixed and variable sampling schemes, which had not yet been studied. They also examined the performance of the proposed approach and showed that it performed as effectively as powerful maximum likelihood estimator-based approaches in some cases and much better than popular fuzzy clustering methods in both fixed and variable sampling control charts. Şentürk and Erginel $^{24}$ introduced the framework of fuzzy $\widetilde{\bar{X}}-\widetilde{R}$ and $\tilde{\bar{X}}$ $\widetilde{S}$ control charts with $\alpha$-cuts by using $\alpha$-level fuzzy midrange. They obtained fuzzy $\widetilde{\bar{X}}-\widetilde{R}$ and $\tilde{\bar{X}}$ $\widetilde{S}$ control charts with triangular fuzzy numbers $(a$, $b$, c). In addition, they developed $\alpha$-cut fuzzy $\widetilde{\bar{X}}$ $\widetilde{R}$ control charts and $\alpha$-cut fuzzy $\tilde{\bar{X}}-\widetilde{S}$ control charts by using an $\alpha$-cut approach. Finally, they calculated $\alpha$-level fuzzy midranges for fuzzy $\widetilde{\bar{X}}-\widetilde{R}$ and $\tilde{\bar{X}}-\tilde{S}$ control charts by using $\alpha$-level fuzzy midrange transformation techniques.

The fuzzy regression control chart was presented by Şentürk ${ }^{25}$. In this paper, fuzzy transformation techniques are represented in Section 2, and the fuzzy $\tilde{\bar{X}}$-regression control chart for $\alpha$-cut based on midrange transformation techniques is given in terms of detailed equations and applications on the fuzzy regression control chart, which are added for evaluating the threading of the inner diameter of a natural gas valve by CNC machines. The main contribution of this study is to give the equations of the fuzzy regression control chart by integrating the traditional regression control chart and fuzzy set theory. Additionally, the fuzzy regression control chart is applied to the tool wearing problem.

Developing the fuzzy regression control chart gives an opportunity for samples to be expressed as fuzzy numbers. The fuzzy upper and lower control limits and center line are defined as fuzzy membership functions in a fuzzy regression control chart. These characteristics provide more accurate decisions on a sample when the sample mean is too close to the control limits and the measurement systems is not very sensitive.
The steps for constructing the fuzzy $\widetilde{\bar{X}}$ regression control chart are described briefly:

Step 1: Data are collected from the process in which the data has approximate values, as in Table 1. By considering the uncertainty due to the nature of process, the measurement system and environmental conditions, data are expressed as triangular fuzzy numbers $(\mathrm{a}, \mathrm{b}, \mathrm{c})$, as in Table 2 . Triangular and trapezoidal membership functions, etc., are used to describe uncertainty.

Step 2: The theoretical structure of the fuzzy $\tilde{\bar{X}}$ regression control chart is developed by using the conventional regression control chart and a fuzzy approach.

Step 3: The $\alpha$-cut fuzzy $\tilde{\bar{X}}$-regression control chart is formulated by integrating an $\alpha$-cut.

Step 4: The $\alpha$-level fuzzy midrange for the $\alpha$-cut fuzzy $\widetilde{\bar{X}}$-regression control chart is derived.

Step 5: Process conditions are set to evaluate the process with the $\alpha$-level fuzzy midrange for the $\alpha$-cut fuzzy $\tilde{\bar{X}}$-regression control chart.

The rest of the paper is organized in the following order. Fuzzy transformation techniques are introduced in Section 2. The theoretical basis of the fuzzy $\tilde{\bar{X}}$-regression control chart is proposed for triangular and trapezoidal membership functions in Section 3. The theoretical structure of a fuzzy $\widetilde{R}$ control chart, developed by Şentürk and Erginel ${ }^{21}$, is given in Section 4. Additionally, the formulation of the fuzzy $\widetilde{R}$ control chart for trapezoidal membership functions is given in this paper. The application to the natural gas valve is given in Section 5. The conclusions are discussed in Section 6.

\section{Fuzzy Transformation Techniques}

Fuzzy transformation techniques are used to transform the fuzzy numbers into crisp values. The four fuzzy measures of central tendency, fuzzy mode, $\alpha$-level fuzzy midrange, fuzzy median and fuzzy average, which well-known in descriptive statistics, are given below ${ }^{8}$ : 
The fuzzy mode, $f_{\text {mode }}$ : The fuzzy mode of a fuzzy set $F$ is the value of the base variable where the membership function equals 1 . This is stated as:

$$
f_{\text {mode }}=\left\{x \mid \mu_{\mathrm{F}}(x)=1\right\}, \quad \forall x \in F .
$$

It is unique if the membership function is unimodal.

The $\alpha$-level fuzzy midrange, $f_{m r}^{\alpha}$ : The average of the end points of an $\alpha$-cut. An $\alpha$-cut, denoted by $F_{\alpha}$, is a non fuzzy subset of the base variable $x$ containing all the values with membership function values greater than or equal to $\alpha$. Thus $F_{\alpha}=\left\{x \mid \mu_{F}(x) \geq \alpha\right\}$. If $a_{\alpha}$ and $c_{\alpha}$ are end points of $\alpha$-cut $\quad F_{\alpha}$ such that $a_{\alpha}=\operatorname{Min}\left\{F_{\alpha}\right\}$ and $c_{\alpha}=\operatorname{Max}\left\{F_{\alpha}\right\}$, then,

$$
f_{m r}^{\alpha}=\frac{1}{2}\left(a_{\alpha}+c_{\alpha}\right)
$$

The fuzzy median, $f_{\text {med }}$ : This is the point that partitions the curve under the membership function of a fuzzy set into two equal regions satisfying the following equation:

$$
\int_{a}^{f_{\text {med }}} \mu_{F}(x) d x=\int_{f_{\text {med }}}^{c} \mu_{F}(x) d x=\frac{1}{2} \int_{a}^{c} \mu_{F}(x) d x
$$

where $a$ and $c$ are the end points in the base variable of the fuzzy set $F$ such that $a<c$.

The fuzzy average, $f_{\text {avg }}$ : Based on $\mathrm{Zadeh}^{7}$, the fuzzy average is:

$$
f_{\text {avg }}=A v(x ; F)=\frac{\int_{x=0}^{1} x \mu_{F}(x) d x}{\int_{x=0}^{1} \mu_{F}(x) d x}
$$

It should be pointed out that there is no theoretical basis supporting any one specifically or the selection between them. In general, the first two methods are easier to calculate than the last two when the membership function is nonlinear. Additionally, the fuzzy mode may lead to biased results when the membership function is extremely asymmetrical. The fuzzy midrange is more flexible because one can choose different levels of membership $(\alpha)$ of interest. If the area under the membership function is considered to be an appropriate measure of fuzziness, the fuzzy median is suitable ${ }^{8}$. The classical control charts are introduced and the theoretical structure of a fuzzy regression control chart is given as the following section.

\section{Fuzzy $\tilde{\bar{X}}$-Regression Control Chart}

When the process data exhibit an underlying trend due to systemic causes, the regression approach is commonly used for monitoring and control. Data describing inventory levels, product yields, productivity ratios, sales figures and tool wear may exhibit linear trends. In this case, instead of using standard Shewhart charts, quality expert implement regression-based control charts are used to monitor a process with a systemic trend, while ordinary least squares regression is commonly used to estimate the trending process mean for these charts ${ }^{1}$.

A regression control chart that integrates linear regression and control chart theory has proven useful in a wide variety of applications, as it requires only a least squares regression equation to process the data prior to constructing the control chart $^{2}$.

The traditional regression control chart was first proposed for the tool wearing problem by Mandel ${ }^{5}$. Mandel set the linear regression $\left(\bar{x}_{j}=\beta_{0}+\beta_{1} t_{j}+\varepsilon\right)$ instead of the general mean $(\overline{\bar{X}})$ of collected data for the related quality characteristic. The parameters of the linear regression model $\left(\beta_{0}, \beta_{1}\right)$ are estimated by using the following normal equations based on the least squares method. Here, a linear system has a special name, such as "normal equations". It is the most direct way of solving a linear squares problem.

$$
\begin{aligned}
& \sum_{j=1}^{m} \bar{x}_{j}=m \beta_{0}+\beta_{1} \sum_{j=1}^{m} t_{j} \\
& \sum_{j=1}^{m} \bar{x}_{j} \cdot t_{j}=\beta_{0} \sum_{j=1}^{m} t_{j}+\beta_{1} \sum_{j=1}^{m} t_{j}^{2}
\end{aligned}
$$

where, $\bar{x}_{j}=\bar{X}_{j}-\overline{\bar{X}}$.

$\bar{X}_{j}$ represents the average of $n$ observations in the $j$ th sample, and $\overline{\bar{X}}$ is the overall mean. $n$ is the sample size, and $t_{j}$ is the number of the $j$ th sample that is related to time. $m$ represents the sample numbers $(j=1,2, \cdots, m) .\left(\hat{\beta}_{0}, \hat{\beta}_{1}\right)$ are estimated from the solutions to the normal equations: 


$$
\begin{gathered}
\hat{\beta}_{0}=\frac{\sum_{j=1}^{m} \bar{x}_{j}-\hat{\beta}_{1} \sum_{j=1}^{m} t_{j}}{m} \\
\hat{\beta}_{1}=\frac{\sum_{j=1}^{m} \bar{x}_{j}\left(t_{j}-\bar{t}\right)}{\sum_{j=1}^{m}\left(t_{j}-\bar{t}\right)^{2}}
\end{gathered}
$$

where $\bar{t}$ is the average of the $t_{i}{ }^{\prime} s$.

Control limits of the traditional regression control chart were defined as $\pm 3 \sigma$ for known variations of populations and $\pm A_{2} \bar{R}$ for variations estimated from samples. The traditional regression control chart for unknown variation is calculated ${ }^{2}$ as:

$$
\begin{aligned}
& U C L_{\operatorname{Re} g-\bar{X}, j}=\hat{\beta}_{0}+\hat{\beta}_{1} t_{j}+A_{2} \bar{R} \\
& C L_{\operatorname{Re} g-\bar{X}, j}=\hat{\beta}_{0}+\hat{\beta}_{1} t_{j} \\
& L C L_{\operatorname{Re} g-\bar{X}, j}=\hat{\beta}_{0}+\hat{\beta}_{1} t_{j}-A_{2} \bar{R}
\end{aligned}
$$

where $A_{2}$ is a control chart coefficient ${ }^{26}, \bar{R}$ is the average of the $R_{i}{ }^{\prime} s$ that are the ranges of samples, and $j=1,2, \ldots, m$.

Given the theoretical structure of the traditional regression control chart mentioned above, the uncertainty and vagueness come from a process and measurement system used for constructing the fuzzy regression control chart. Another reason for constructing the regression control chart is to consider the spread of the dependent and independent variables. However, in the conventional regression control chart based on the numerical data only, some useful insights and valuable information may be lost. The approximate values for a sample or subgroup are represented by using triangular fuzzy numbers $(a, b, c$,$) or$ trapezoidal fuzzy numbers $(a, b, c, d)$ in the fuzzy case. Although there are many membership functions, such as Gaussian or generalized membership functions, etc, the theoretical structure of fuzzy regression charts for triangular (in Section 3.1) and trapezoidal (in Section 3.2) membership functions is presented in this paper.

\subsection{Fuzzy $\tilde{\bar{X}}$-regression control chart for TFNs case}

Data are represented as fuzzy numbers as $\left(X_{a, i j}, X_{b, i j}, X_{c, i j}\right)$ according to triangular membership function parameters $(a, b, c)$, and the fuzzy averages of each sample $\left(\bar{X}_{a, j}, \bar{X}_{b, j}, \bar{X}_{c, j}\right)$ are calculated as follows:

$$
\begin{gathered}
\bar{X}_{a, j}=\frac{\sum_{i=1}^{n} X_{a, i j}}{n} \\
\bar{X}_{b, j}=\frac{\sum_{i=1}^{n} X_{b, i j}}{n} \\
\bar{X}_{c, j}=\frac{\sum_{i=1}^{n} X_{c, i j}}{n}
\end{gathered}
$$

where $i=1,2, \ldots ., n$ and $j=1,2, \ldots ., m$.

The fuzzy linear regression model for the average of each fuzzy number can be estimated by the following equations:

$$
\begin{aligned}
& \bar{X}_{\operatorname{Re} g-a, j}=\hat{\beta}_{0 a}+\hat{\beta}_{1 a} t_{j}+\varepsilon \\
& \bar{X}_{\operatorname{Re} g-b, j}=\hat{\beta}_{0 b}+\hat{\beta}_{1 b} t_{j}+\varepsilon \\
& \bar{X}_{\operatorname{Re} g-c, j}=\hat{\beta}_{0 c}+\hat{\beta}_{1 c} t_{j}+\varepsilon
\end{aligned}
$$

The parameters of the fuzzy linear regression model $\left(\beta_{0 a}, \beta_{1 a}\right)$ are estimated by using the normal equations based on the least squares method for the $\bar{X}_{a, j}{ }^{\prime} s$ as in "Eq. (18-19)". $\left(\hat{\beta}_{0 b}, \hat{\beta}_{1 b}\right)$ and $\left(\hat{\beta}_{0 c}, \hat{\beta}_{1 c}\right)$ are estimated with similar calculations for the $\bar{X}_{b, j}$ 's and $\bar{X}_{c, j}$ 's, respectively.

$$
\begin{gathered}
\hat{\beta}_{0 a}=\frac{\sum_{j=1}^{m} \bar{x}_{a, j}-\hat{\beta}_{1 a} \sum_{j=1}^{m} t_{j}}{m} \\
\hat{\beta}_{1 a}=\frac{\sum_{j=1}^{m} \bar{x}_{a, j}\left(t_{j}-\bar{t}\right)}{\sum_{j=1}^{m}\left(t_{j}-\bar{t}\right)^{2}}
\end{gathered}
$$


where $\bar{x}_{a, j}=\bar{X}_{a, j}-\overline{\bar{X}}_{a}, j=1,2, \ldots ., m$.

$$
\begin{gathered}
\overline{\bar{X}}_{a}=\frac{\sum_{j=1}^{m} \bar{X}_{a, j}}{m} \\
\overline{\bar{X}}_{b}=\frac{\sum_{j=1}^{m} \bar{X}_{b, j}}{m} \\
\overline{\bar{X}}_{c}=\frac{\sum_{j=1}^{m} \bar{X}_{c, j}}{m}
\end{gathered}
$$

$R_{a, j}, R_{b, j}, R_{c, j}$ are the ranges of the $j$ th sample, and $\bar{R}_{a}, \bar{R}_{b}$, and $\bar{R}_{c}$ are the arithmetic means of the least possible values, the most possible values, and the largest possible values by using the ranking method, respectively. Firstly, $R_{a, j}, R_{b, j}, R_{c, j}$ are calculated as follows:

$$
\begin{aligned}
& R_{a, j}=X_{\max , a, j}-X_{\min , c, j} \\
& R_{b, j}=X_{\max , b, j}-X_{\min , b, j} \\
& R_{c, j}=X_{\max , c, j}-X_{\min , a, j}
\end{aligned}
$$

where $\quad\left(X_{\max , a, j}, X_{\max , b, j}, X_{\max , c, j}\right) \quad$ are the maximum fuzzy numbers in the sample, and $\left(X_{\min , a, j}, X_{\min , b, j}, X_{\min , c, j}\right)$ are the minimum fuzzy numbers in the sample. Then,

$$
\begin{gathered}
\bar{R}_{a}=\frac{\sum_{j=1}^{m} R_{a, j}}{m} \\
\bar{R}_{b}=\frac{\sum_{j=1}^{m} R_{b, j}}{m} \\
\bar{R}_{c}=\frac{\sum_{j=1}^{m} R_{c, j}}{m}
\end{gathered}
$$

The theoretical structures of the fuzzy $\tilde{\bar{X}}$ regression control chart can be obtained by the following equations;

$$
\begin{aligned}
& U \widetilde{C} L_{\operatorname{Re} g-\bar{X}, j}=\left(\hat{\beta}_{0 a}+\hat{\beta}_{1 a} t_{j}+A_{2} \bar{R}_{a},\right. \\
& \left.\hat{\beta}_{0 b}+\hat{\beta}_{1 b} t_{j}+A_{2} \bar{R}_{b}, \hat{\beta}_{0 c}+\hat{\beta}_{1 c} t_{j}+A_{2} \bar{R}_{c}\right) \\
& C \widetilde{L}_{\operatorname{Re} g-\bar{X}, j}=\left(\hat{\beta}_{0 a}+\hat{\beta}_{1 a} t_{j},\right. \\
& \left.\hat{\beta}_{0 b}+\hat{\beta}_{1 b} t_{j}, \hat{\beta}_{0 c}+\hat{\beta}_{1 c} t_{j}\right) \\
& L \widetilde{C} L_{\operatorname{Re} g-\bar{X}, j}=\left(\hat{\beta}_{0 a}+\hat{\beta}_{1 a} t_{j}-A_{2} \bar{R}_{c},\right. \\
& \left.\hat{\beta}_{0 b}+\hat{\beta}_{1 b} t_{j}-A_{2} \bar{R}_{b}, \hat{\beta}_{0 c}+\hat{\beta}_{1 c} t_{j}-A_{2} \bar{R}_{a}\right)
\end{aligned}
$$

\subsubsection{The $\alpha$-cut fuzzy $\tilde{\bar{X}}$-regression control chart for TFNs}

An $\alpha$-cut comprises all elements whose membership degrees are greater than equal to $\alpha$. The set $A_{\alpha}=\left\{x \in X: \mu_{A}(x) \geq \alpha, 0 \leq \alpha \leq 1\right\}$.The " $\alpha$-level sets" $A_{\alpha}$ are also called the " $\alpha$-cut sets" ${ }^{, 27,28}$.

For simplicity, in this paper both $\alpha$-level and $\alpha$-cut terms are used with the same meaning. The most often used membership function to describe the uncertainty in model parameters is TFN. Figure 3 shows a triangular fuzzy parameter and its $\alpha$ cuts.

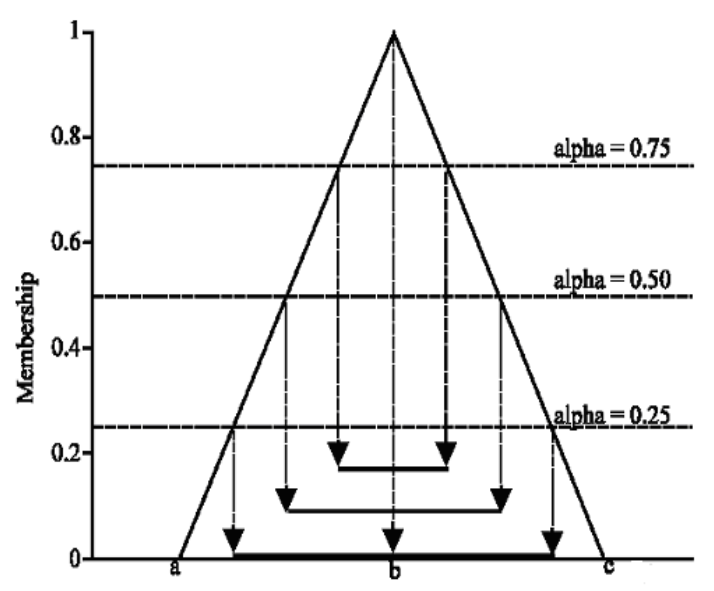

Fig 3. Triangular fuzzy parameter and its $\alpha$-cuts.

Figure 3 also shows that the higher the value, the higher the confidence in the fuzzy parameter ${ }^{27}$.

Applying an $\alpha$-cut to fuzzy set, the values of $\bar{X}_{\text {Re } g-a, j}^{\alpha}, \bar{X}_{\text {Re } g-c, j}^{\alpha}$ and $\bar{R}_{a}^{\alpha}, \bar{R}_{c}^{\alpha}$ are determined as follows:

$$
\begin{aligned}
& \bar{X}_{\operatorname{Re} g-a, j}^{\alpha}=\bar{X}_{\operatorname{Re} g-a, j}+\alpha\left(\bar{X}_{\operatorname{Re} g-b, j}-\bar{X}_{\operatorname{Re} g-a, j}\right) \\
& =\left(\hat{\beta}_{0 a}+\hat{\beta}_{1 a} t_{j}\right)+\alpha\left[\left(\hat{\beta}_{0 b}+\hat{\beta}_{1 b} t_{j}\right)-\left(\hat{\beta}_{0 a}+\hat{\beta}_{1 a} t_{j}\right)\right] \\
& =(1-\alpha)\left(\hat{\beta}_{0 a}-\hat{\beta}_{1 a} t_{j}\right)+\alpha\left(\hat{\beta}_{0 b}+\hat{\beta}_{1 b} t_{j}\right)
\end{aligned}
$$


$\bar{X}_{\operatorname{Re} g-c, j}^{\alpha}=\bar{X}_{\operatorname{Re} g-c, j}-\alpha\left(\bar{X}_{\operatorname{Re} g-c, j}-\bar{X}_{\operatorname{Re} g-b, j}\right)$

$=\left(\hat{\beta}_{0 c}+\hat{\beta}_{1 c} t_{j}\right)-\alpha\left[\left(\hat{\beta}_{0 c}+\hat{\beta}_{1 c} t_{j}\right)-\left(\hat{\beta}_{0 b}+\hat{\beta}_{1 b} t_{j}\right)\right]$

$=(1-\alpha)\left(\hat{\beta}_{0 c}-\hat{\beta}_{1 c} t_{j}\right)+\alpha\left(\hat{\beta}_{0 b}+\hat{\beta}_{1 b} t_{j}\right)$

$\bar{R}_{a}^{\alpha}=\bar{R}_{a}+\alpha\left(\bar{R}_{b}-\bar{R}_{a}\right)$

$\bar{R}_{c}^{\alpha}=\bar{R}_{c}-\alpha\left(\bar{R}_{c}-\bar{R}_{b}\right)$

In an $\alpha$-cut fuzzy $\widetilde{\bar{X}}$-regression control chart, control limits based on ranges can be stated in the following equations:

$$
\begin{aligned}
& U \widetilde{C} L_{\operatorname{Re} g-\bar{X}, j}^{\alpha}=\left(\bar{X}_{\operatorname{Re} g-a, j}^{\alpha}+A_{2} \bar{R}_{a}^{\alpha},\right. \\
& \left.\bar{X}_{\operatorname{Re} g-b, j}+A_{2} \bar{R}_{b}, \quad \bar{X}_{\operatorname{Re} g-c, j}^{\alpha}+A_{2} \bar{R}_{c}^{\alpha}\right) \\
& \widetilde{C} L_{\operatorname{Re} g-\bar{X}, j}^{\alpha}=\left(\bar{X}_{\operatorname{Re} g-a, j}^{\alpha}, \bar{X}_{\operatorname{Re} g-b, j}, \bar{X}_{\operatorname{Re} g-c, j}^{\alpha}\right) \\
& L \widetilde{C} L_{\operatorname{Re} g-\bar{X}, j}^{\alpha}=\left(\bar{X}_{\operatorname{Re} g-a, j}^{\alpha}-A_{2} \bar{R}_{c}^{\alpha}, \bar{X}_{\operatorname{Re} g-b, j}-A_{2} \bar{R}_{b},\right. \\
& \left.\quad \bar{X}_{\operatorname{Re} g-c, j}^{\alpha}-A_{2} \bar{R}_{a}^{\alpha}\right)
\end{aligned}
$$

3.1.2. $\alpha$-cut fuzzy $\tilde{\bar{X}}$-regression control chart for TFNs based on $\alpha$-level fuzzy midrange transformation

Fuzzy transformation techniques are used for deciding if the process is "under-control" or "outof-control" after calculating the control limits. The fuzzy linear regression model can be transformed to crisp numbers with the fuzzy transformation techniques. In this paper, the fuzzy midrange transformation technique is used. The $\alpha$-level fuzzy midrange, $f_{m r}^{\alpha}$, is defined in "Eq. (2)". The $\alpha$-level fuzzy midrange of sample $j, S_{m r, j}^{\alpha}$, is determined by:

$$
S_{m r, j}^{\alpha}=\frac{\left(a_{j}+c_{j}\right)+\alpha\left[\left(b_{j}-a_{j}\right)-\left(c_{j}-b_{j}\right)\right]}{2}
$$

The $\alpha$-level fuzzy midrange for an $\alpha$-cut fuzzy $\widetilde{\bar{X}}$-regression control chart can be calculated by using the midrange transformation technique as follows:

$$
U C C_{m r-\operatorname{Re} g-\bar{X}, j}^{\alpha}=\frac{\left(\bar{X}_{\mathrm{Re} g-a, j}^{\alpha}+\bar{X}_{\mathrm{Re} g-c, j}^{\alpha}\right)}{2}+A_{2}\left(\frac{\bar{R}_{a}^{\alpha}+\bar{R}_{c}^{\alpha}}{2}\right)
$$

$$
\begin{aligned}
& C L_{m r-\operatorname{Re} g-\bar{X}, j}^{\alpha}=\frac{\left(\bar{X}_{\operatorname{Re} g-a, j}^{\alpha}+\bar{X}_{\operatorname{Re} g-c, j}^{\alpha}\right)}{2} \\
& L C L_{m r-\operatorname{Re} g-\bar{X}, j}^{\alpha}=\frac{\left(\bar{X}_{\operatorname{Re} g-a, j}^{\alpha}+\bar{X}_{\operatorname{Re} g-c, j}^{\alpha}\right)}{2}-A_{2}\left(\frac{\bar{R}_{a}^{\alpha}+\bar{R}_{c}^{\alpha}}{2}\right)
\end{aligned}
$$

The fuzzy midrange of $j$ th sample is:

$$
\begin{aligned}
& S_{m r-\operatorname{Re} g-\bar{X}, j}^{\alpha}=\frac{\left(\bar{X}_{\operatorname{Re} g-a, j}+\bar{X}_{\operatorname{Re} g-c, j}\right)}{2}+ \\
& \frac{\alpha\left[\left(\bar{X}_{\operatorname{Re} g-b, j}-\bar{X}_{\operatorname{Re} g-a, j}\right)-\left(\bar{X}_{\operatorname{Re} g-c, j}-\bar{X}_{\operatorname{Re} g-b, j}\right)\right]}{2}
\end{aligned}
$$

The condition of process control for each sample can be defined $\mathrm{as}^{3}$ :

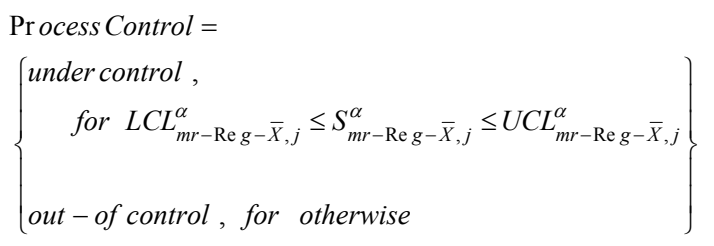

\subsection{Fuzzy $\widetilde{\bar{X}}$-regression control chart for TrFNs case}

The fuzzy averages of each sample $\left(\bar{X}_{a, j}, \bar{X}_{b, j}, \bar{X}_{c, j}, \bar{X}_{d, j}\right)$ are calculated based on $\operatorname{TrFNs}\left(X_{a, i j}, X_{b, i j}, X_{c, i j}, X_{d, i j}\right)$ as follows:

$$
\begin{aligned}
& \bar{X}_{a, j}=\frac{\sum_{i=1}^{n} X_{a, i j}}{n} \\
& \bar{X}_{b, j}=\frac{\sum_{i=1}^{n} X_{b, i j}}{n} \\
& \bar{X}_{c, j}=\frac{\sum_{i=1}^{n} X_{c, i j}}{n} \\
& \bar{X}_{d, j}=\frac{\sum_{i=1}^{n} X_{d, i j}}{n}
\end{aligned}
$$

where $i=1,2, \ldots, n$ and $j=1,2, \ldots, m$.

The fuzzy linear regression model for the average of each fuzzy trapezoidal number can be estimated by using the following: 


$$
\begin{aligned}
& \bar{X}_{\mathrm{Re} g-a, j}=\hat{\beta}_{0 a}+\hat{\beta}_{1 a} t_{j}+\varepsilon \\
& \bar{X}_{\operatorname{Re} g-b, j}=\hat{\beta}_{0 b}+\hat{\beta}_{1 b} t_{j}+\varepsilon \\
& \bar{X}_{\operatorname{Re} g-c, j}=\hat{\beta}_{0 c}+\hat{\beta}_{1 c} t_{j}+\varepsilon \\
& \bar{X}_{\operatorname{Re}-d, j}=\hat{\beta}_{0 d}+\hat{\beta}_{1 d} t_{j}+\varepsilon
\end{aligned}
$$

The parameters of the fuzzy linear regression model $\left(\beta_{0}, \beta_{1}\right)$ are estimated for $(\mathrm{a}, \mathrm{b}, \mathrm{c}, \mathrm{d})$ as in "Eq. (18-19)".

The overall mean for fuzzy number $(a, b, c, d)$ is calculated as follows:

$$
\begin{aligned}
& \overline{\bar{X}}_{a}=\frac{\sum_{j=1}^{m} \bar{X}_{a, j}}{m} \\
& \overline{\bar{X}}_{b}=\frac{\sum_{j=1}^{m} \bar{X}_{b, j}}{m} \\
& \overline{\bar{X}}_{c}=\frac{\sum_{j=1}^{m} \bar{X}_{c, j}}{m} \\
& \overline{\bar{X}}_{d}=\frac{\sum_{j=1}^{m} \bar{X}_{d, j}}{m}
\end{aligned}
$$

$R_{a, j}, R_{b, j}, R_{c, j}, R_{d, j}$ are the ranges of the $j$ th sample, and their averages are estimated as follows:

$$
\begin{aligned}
& R_{a, j}=X_{\max , a, j}-X_{\min , d, j} \\
& R_{b, j}=X_{\max , b, j}-X_{\min , c, j} \\
& R_{c, j}=X_{\max , c, j}-X_{\min , b, j} \\
& R_{c, j}=X_{\max , d, j}-X_{\min , a, j} \\
& \bar{R}_{a}=\frac{\sum_{j=1}^{m} R_{a, j}}{m} \\
& \bar{R}_{b}=\frac{\sum_{j=1}^{m} R_{b, j}}{m}
\end{aligned}
$$

$$
\begin{gathered}
\bar{R}_{c}=\frac{\sum_{j=1}^{m} R_{c, j}}{m} \\
\bar{R}_{d}=\frac{\sum_{j=1}^{m} R_{d, j}}{m}
\end{gathered}
$$

The formulation of the fuzzy $\tilde{\bar{X}}$ regression control chart for TrFNs can be obtained as follows:

$$
\begin{aligned}
& U \widetilde{C} L_{\operatorname{Re} g-\bar{X}, j}= \\
& \left(\hat{\beta}_{0 a}+\hat{\beta}_{1 a} t_{j}+A_{2} \bar{R}_{a}, \hat{\beta}_{0 b}+\hat{\beta}_{1 b} t_{j}+A_{2} \bar{R}_{b},\right. \\
& \left.\hat{\beta}_{0 c}+\hat{\beta}_{1 c} t_{j}+A_{2} \bar{R}_{c,} \hat{\beta}_{0 d}+\hat{\beta}_{1 d} t_{j}+A_{2} \bar{R}_{d}\right) \\
& C \widetilde{L}_{\operatorname{Re} g-\bar{X}, j}=\left(\hat{\beta}_{0 a}+\hat{\beta}_{1 a} t_{j},\right. \\
& \hat{\beta}_{0 b}+\hat{\beta}_{1 b} t_{j}, \hat{\beta}_{0 c}+\hat{\beta}_{1 c} t_{j}, \\
& \left.\hat{\beta}_{0 d}+\hat{\beta}_{1 d} t_{j}\right) \\
& L \widetilde{C} L_{\operatorname{Re} g-\bar{X}, j}=\left(\hat{\beta}_{0 a}+\hat{\beta}_{1 a} t_{j}-A_{2} \bar{R}_{d},\right. \\
& \hat{\beta}_{0 b}+\hat{\beta}_{1 b} t_{j}-A_{2} \bar{R}_{c}, \hat{\beta}_{0 c}+\hat{\beta}_{1 c} t_{j}-A_{2} \bar{R}_{b}, \\
& \left.\hat{\beta}_{0 d}+\hat{\beta}_{1 d} t_{j}-A_{2} \bar{R}_{a}\right)
\end{aligned}
$$

3.2.1. The $\alpha$-cut fuzzy $\tilde{\bar{X}}$-regression control chart for TrFNs

Applying the $\alpha$-cut to a fuzzy set, the values of $\bar{X}_{\operatorname{Re} g-a, j}^{\alpha}, \bar{X}_{\operatorname{Re} g-d, j}^{\alpha}$ and $\bar{R}_{a}^{\alpha}, \bar{R}_{d}^{\alpha}$ are determined as follows:

$$
\begin{aligned}
& \bar{X}_{\operatorname{Re} g-a, j}^{\alpha}=\bar{X}_{\operatorname{Re} g-a, j}+\alpha\left(\bar{X}_{\operatorname{Re} g-b, j}-\bar{X}_{\operatorname{Re} g-a, j}\right) \\
& =\left(\hat{\beta}_{0 a}+\hat{\beta}_{1 a} t_{j}\right)+\alpha\left[\left(\hat{\beta}_{0 b}+\hat{\beta}_{1 b} t_{j}\right)-\left(\hat{\beta}_{0 a}+\hat{\beta}_{1 a} t_{j}\right)\right] \\
& =(1-\alpha)\left(\hat{\beta}_{0 a}-\hat{\beta}_{1 a} t_{j}\right)+\alpha\left(\hat{\beta}_{0 b}+\hat{\beta}_{1 b} t_{j}\right) \\
& \bar{X}_{\operatorname{Re} g-d, j}^{\alpha}=\bar{X}_{\operatorname{Re} g-d, j}-\alpha\left(\bar{X}_{\operatorname{Re} g-d, j}-\bar{X}_{\operatorname{Re} g-c, j}\right) \\
& =\left(\hat{\beta}_{0 d}+\hat{\beta}_{1 d} t_{j}\right)-\alpha\left[\left(\hat{\beta}_{0 d}+\hat{\beta}_{1 d} t_{j}\right)-\left(\hat{\beta}_{0 c}+\hat{\beta}_{1 c} t_{j}\right)\right] \\
& =(1-\alpha)\left(\hat{\beta}_{0 d}-\hat{\beta}_{1 d} t_{j}\right)+\alpha\left(\hat{\beta}_{0 c}+\hat{\beta}_{1 c} t_{j}\right)
\end{aligned}
$$

$$
\begin{aligned}
& \bar{R}_{a}^{\alpha}=\bar{R}_{a}+\alpha\left(\bar{R}_{b}-\bar{R}_{a}\right) \\
& \bar{R}_{d}^{\alpha}=\bar{R}_{d}-\alpha\left(\bar{R}_{d}-\bar{R}_{c}\right)
\end{aligned}
$$

The formulations of a fuzzy $\tilde{\bar{X}}$ regression control chart for TrFNs with an $\alpha$-cut are given as follows: 


$$
\begin{aligned}
& U \widetilde{C} L_{\operatorname{Re} g-\bar{X}, j}^{\alpha}=\left(\bar{X}_{\operatorname{Re} g-a, j}^{\alpha}+A_{2} \bar{R}_{a}^{\alpha},\right. \\
& \bar{X}_{\operatorname{Re} g-b, j}+A_{2} \bar{R}_{b}, \quad \bar{X}_{\operatorname{Re} g-c, j}+A_{2} \bar{R}_{c}, \\
& \left.\bar{X}_{\operatorname{Re} g-d, j}^{\alpha}+A_{2} \bar{R}_{d}^{\alpha}\right) \\
& \widetilde{C} L_{\operatorname{Re} g-\bar{X}, j}^{\alpha}=\left(\bar{X}_{\operatorname{Re} g-a, j}^{\alpha}, \bar{X}_{\operatorname{Re} g-b, j},\right. \\
& \left.\bar{X}_{\operatorname{Re} g-c, j}, \bar{X}_{\operatorname{Re} g-d, j}^{\alpha}\right) \\
& L \widetilde{C}_{\operatorname{Re} g-\bar{X}, j}^{\alpha}=\left(\bar{X}_{\operatorname{Re} g-a, j}^{\alpha}-A_{2} \bar{R}_{d}^{\alpha},\right. \\
& \bar{X}_{\operatorname{Re} g-b, j}-A_{2} \bar{R}_{c}, \quad \bar{X}_{\operatorname{Re} g-c, j}-A_{2} \bar{R}_{b}, \\
& \left.\bar{X}_{\operatorname{Re} g-d, j}^{\alpha}-A_{2} \bar{R}_{a}^{\alpha}\right)
\end{aligned}
$$

3.2.2. The $\alpha$-cut fuzzy $\widetilde{\bar{X}}$-regression control chart for TrFNs based on an a-level fuzzy midrange transformation

The $\alpha$-level fuzzy midrange of sample $j, S_{m r, j}^{\alpha}$, is determined as follows:

$$
S_{m r, j}^{\alpha}=\frac{\left(a_{j}+d_{j}\right)+\alpha\left[\left(b_{j}-a_{j}\right)-\left(d_{j}-c_{j}\right)\right]}{2}
$$

The $\alpha$-level fuzzy midrange for the $\alpha$-cut fuzzy $\widetilde{\bar{X}}$-regression control chart for TrFNs can be calculated by using the midrange transformation technique:

$$
\begin{aligned}
& U C L_{m r-\operatorname{Re} g-\bar{X}, j}^{\alpha}= \\
& \frac{\left(\bar{X}_{\operatorname{Re} g-a, j}^{\alpha}+\bar{X}_{\operatorname{Re} g-d, j}^{\alpha}\right)}{2}+A_{2}\left(\frac{\bar{R}_{a}^{\alpha}+\bar{R}_{d}^{\alpha}}{2}\right) \\
& C L_{m r-\operatorname{Re} g-\bar{X}, j}^{\alpha}=\frac{\left(\bar{X}_{\operatorname{Re} g-a, j}^{\alpha}+\bar{X}_{\operatorname{Re} g-d, j}^{\alpha}\right)}{2} \\
& L C L_{m r-\operatorname{Re} g-\bar{X}, j}^{\alpha}= \\
& \frac{\left(\bar{X}_{\operatorname{Re} g-a, j}^{\alpha}+\bar{X}_{\operatorname{Re} g-d, j}^{\alpha}\right)}{2}-A_{2}\left(\frac{\bar{R}_{a}^{\alpha}+\bar{R}_{d}^{\alpha}}{2}\right)
\end{aligned}
$$

The fuzzy midrange of the $j$ th sample is calculated by using Eq. (79):

$$
\begin{aligned}
& S_{m r-\operatorname{Re} g-\bar{X}, j}^{\alpha}=\frac{\left(\bar{X}_{\operatorname{Re} g-a, j}+\bar{X}_{\operatorname{Re} g-c, j}\right)}{2}+ \\
& \frac{\alpha\left[\left(\bar{X}_{\operatorname{Re} g-b, j}-\bar{X}_{\operatorname{Re} g-a, j}\right)-\left(\bar{X}_{\operatorname{Re} g-d, j}-\bar{X}_{\operatorname{Re} g-c, j}\right)\right]}{2}
\end{aligned}
$$

The condition of process control for each sample can be defined as $^{3}$ :

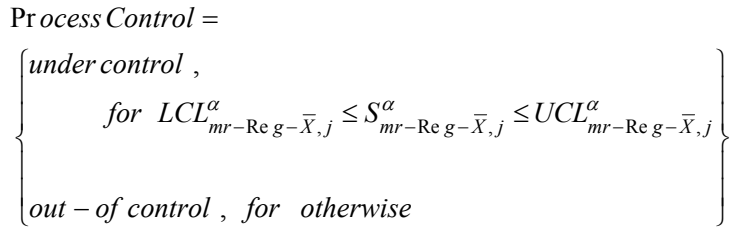

\section{Fuzzy $\widetilde{\mathbf{R}}$ Control Chart}

The $\mathrm{R}$ control chart is used together with the regression control chart to evaluate the deviation when the sample size smaller then $10(n<10)$. If $\mathrm{n}>10$, the $S$ control chart should be used for evaluating and monitoring the process variability .Fuzzy $\widetilde{R}$ control charts for TFNs and TrFNs are detailed in Section 4.1 and Section 4.2, respectively.

\subsection{Fuzzy $\widetilde{R}$ control chart for TFNs case}

Fuzzy $\widetilde{R}$ control chart limits are obtained with triangular fuzzy numbers. The $\alpha$-level fuzzy midrange for the $\alpha$-cut fuzzy $\widetilde{R}$ control chart was introduced in the following equations “Eq.'s. (8191)" by Şentürk and Erginel ${ }^{24}$ :

$$
\begin{aligned}
& U \widetilde{C} L_{R}=D_{4}\left(\bar{R}_{a}, \bar{R}_{b}, \bar{R}_{c}\right) \\
& C \widetilde{L}_{R}=\left(\bar{R}_{a}, \bar{R}_{b}, \bar{R}_{c}\right) \\
& L \widetilde{C} L_{R}=D_{3}\left(\bar{R}_{a}, \bar{R}_{b}, \bar{R}_{c}\right)
\end{aligned}
$$

where $D_{4}$ and $D_{3}$ are the control chart coefficients ${ }^{26}$.

\subsubsection{The $\alpha$-cut fuzzy $\widetilde{R}$ control chart for TFNs}

Control limits of the $\alpha$-cut fuzzy $\widetilde{R}$ control chart can be stated as follows:

$$
\begin{aligned}
& U \widetilde{C} L_{R}^{\alpha}=D_{4}\left(\bar{R}_{a}^{\alpha}, \bar{R}_{b}, \bar{R}_{c}^{\alpha}\right) \\
& C \widetilde{L}_{R}^{\alpha}=\left(\bar{R}_{a}^{\alpha}, \bar{R}_{b}, \bar{R}_{c}^{\alpha}\right) \\
& L \widetilde{C} R_{R}^{\alpha}=D_{3}\left(\bar{R}_{a}^{\alpha}, \bar{R}_{b}, \bar{R}_{c}^{\alpha}\right)
\end{aligned}
$$

4.1.2 The $\alpha$-cut fuzzy $\widetilde{R}$ control chart for TFNs based on an $\alpha$-level fuzzy midrange transformation

Control limits of the $\alpha$-level fuzzy midrange for the $\alpha$-cut fuzzy $\widetilde{R}$ control chart can be calculated as follows:

$$
U C L_{m r-R}^{\alpha}=D_{4} f_{m r-R}^{\alpha}(C \tilde{L})
$$




$$
\begin{aligned}
& C L_{m r-R}^{\alpha}=f_{m r-R}^{\alpha}(C \widetilde{L})=\frac{\bar{R}_{a}^{\alpha}+\bar{R}_{c}^{\alpha}}{2} \\
& L C L_{m r-R}^{\alpha}=D_{3} f_{m r-R}^{\alpha}(C \tilde{L})
\end{aligned}
$$

The definition of an $\alpha$-level fuzzy midrange of sample $j$ for fuzzy $\widetilde{R}$ control chart is:

$S_{m r-R, j}^{\alpha}=$
$\frac{\left(R_{a, j}+R_{c, j}\right)+\alpha\left[\left(R_{b, j}-R_{a, j}\right)-\left(R_{c, j}-R_{b, j}\right)\right]}{2}$

The condition of process control for each sample can be defined as:

Process Control $=\left\{\begin{array}{l}\text { under control }, \\ \quad \text { for } L C L_{m r-R}^{\alpha} \leq S_{m r-R, j}^{\alpha} \leq U C L_{m r-R}^{\alpha} \\ \text { out }- \text { of control, for otherwise }\end{array}\right\}$

\subsection{Fuzzy $\widetilde{\mathbf{R}}$ control chart for TrFNs case}

The formulation of the fuzzy $\widetilde{R}$ control chart based on $\operatorname{TrFN}$ are given as follows:

$$
\begin{aligned}
& U \widetilde{C} L_{R}=D_{4}\left(\bar{R}_{a}, \bar{R}_{b}, \bar{R}_{c}, \bar{R}_{d}\right) \\
& C \widetilde{L}_{R}=\left(\bar{R}_{a}, \bar{R}_{b}, \bar{R}_{c}, \bar{R}_{d}\right) \\
& L \widetilde{C} L_{R}=D_{3}\left(\bar{R}_{a}, \bar{R}_{b}, \bar{R}_{c}, \bar{R}_{d}\right)
\end{aligned}
$$

\subsubsection{The $\alpha$-cut fuzzy $\widetilde{\mathrm{R}}$ control chart for TrFNs}

Control limits of an $\alpha$-cut fuzzy $\widetilde{R}$ control chart based on $\mathrm{TrFN}$ are given as follows:

$$
\begin{aligned}
& U \widetilde{C} L_{R}^{\alpha}=D_{4}\left(\bar{R}_{a}^{\alpha}, \bar{R}_{b}, \bar{R}_{c}, \bar{R}_{d}^{\alpha}\right) \\
& C \widetilde{L}_{R}^{\alpha}=\left(\bar{R}_{a}^{\alpha}, \bar{R}_{b}, \bar{R}_{c}, \bar{R}_{d}^{\alpha}\right) \\
& L \widetilde{C} R_{R}^{\alpha}=D_{3}\left(\bar{R}_{a}^{\alpha}, \bar{R}_{b}, \bar{R}_{c}, \bar{R}_{d}^{\alpha}\right)
\end{aligned}
$$

4.2.2 The $\alpha$-cut fuzzy $\widetilde{R}$ control chart for TrFNs based on an $\alpha$-level fuzzy midrange transformation

The formulation of control limits of an $\alpha$-level fuzzy midrange for an $\alpha$-cut fuzzy $\widetilde{R}$ control chart for $\operatorname{TrFN}$ can be calculated as follows:

$$
\begin{aligned}
& U C L_{m r-R}^{\alpha}=D_{4} f_{m r-R}^{\alpha}(C \widetilde{L}) \\
& C L_{m r-R}^{\alpha}=f_{m r-R}^{\alpha}(C \tilde{L})=\frac{\bar{R}_{a}^{\alpha}+\bar{R}_{d}^{\alpha}}{2}
\end{aligned}
$$

$$
L C L_{m r-R}^{\alpha}=D_{3} f_{m r-R}^{\alpha}(C \tilde{L})
$$

The definition of $\alpha$-level fuzzy midrange of sample $j$ for fuzzy $\widetilde{R}$ control chart calculated as follows:

$$
\begin{aligned}
& S_{m r-R, j}^{\alpha}= \\
& \frac{\left(R_{a, j}+R_{d, j}\right)+\alpha\left[\left(R_{b, j}-R_{a, j}\right)-\left(R_{d, j}-R_{c, j}\right)\right]}{2}
\end{aligned}
$$

The condition of process control for each sample can be defined as:

$$
\text { Process Control }=\left\{\begin{array}{c}
\text { under control , } \\
\text { for } L C L_{m r-R}^{\alpha} \leq S_{m r-R, j}^{\alpha} \leq U C L_{m r-R}^{\alpha} \\
\text { out }- \text { of control, for otherwise }
\end{array}\right\}
$$

\section{An Application for Fuzzy $\widetilde{\bar{X}}$-Regression and $\widetilde{R}$ Control Charts}

The threading of the natural gas valve by $\mathrm{CNC}$ machines was used an example application. An insert was used as a tool to thread parts. After a certain number of natural gas valves are threaded, the insert should be changed to a new one because of the tool wearing problem. From the manufacturing process, inner diameters of natural gas valves were measured as approximate values, as in Table 1. Data were converted to triangular fuzzy numbers $(\mathrm{a}, \mathrm{b}, \mathrm{c})$ in Table 2. The regression equations of the application were calculated as in "Eq's. (109-111)" because the data has a trend. These regression equations were used instead of the average. The equations for the " $\alpha$-level fuzzy midrange for $\alpha$-cut fuzzy $\widetilde{\bar{X}}$-regression control chart" and the " $\alpha$-level fuzzy midrange for $\alpha$-cut fuzzy $\widetilde{R}$ control chart" for the fuzzy inner diameter of natural gas valves were evaluated by the following steps:

Step 1: Twenty-five samples with 5 sample sizes were collected from the manufacturing process as an approximate value throughout a certain time period, as shown in Table 1. These values are represented by triangular fuzzy numbers $\left(X_{a, i j}, X_{b, i j}, X_{c, i j}\right)$, as shown in Table 2. 
Table 1. The approximate values of inner diameters of natural gas valves.

\begin{tabular}{|c|c|}
\hline Time & Approximately \\
\hline $\mathbf{1}$ & 1.41 \\
\hline & 1.40 \\
\hline & 1.40 \\
\hline & 1.40 \\
\hline & 1.45 \\
\hline $\mathbf{2}$ & 1.43 \\
\hline & 1.41 \\
\hline & 1.43 \\
\hline & 1.44 \\
\hline & 1.46 \\
\hline$\vdots$ & $\vdots$ \\
\hline $\mathbf{2 5}$ & 1.45 \\
\hline & 1.50 \\
\hline & 1.50 \\
\hline & 1.50 \\
\hline & 1.50 \\
\hline
\end{tabular}

Table 2. Fuzzy triangular number $(a, b, c)$ representation of 25 samples.

\begin{tabular}{|c|c|c|c|}
\hline Time & $\mathbf{a}$ & $\mathbf{b}$ & $\mathbf{c}$ \\
\hline 1 & 1.35 & 1.41 & 1.45 \\
\hline & 1.32 & 1.40 & 1.41 \\
\hline & 1.34 & 1.40 & 1.44 \\
\hline & 1.33 & 1.40 & 1.48 \\
\hline & 1.43 & 1.45 & 1.50 \\
\hline $\mathbf{2}$ & 1.37 & 1.43 & 1.48 \\
\hline & 1.31 & 1.41 & 1.43 \\
\hline & 1.33 & 1.43 & 1.45 \\
\hline & 1.34 & 1.44 & 1.46 \\
\hline & 1.44 & 1.46 & 1.49 \\
\hline$\vdots$ & $\vdots$ & $\vdots$ & $\vdots$ \\
\hline $\mathbf{2 5}$ & 1.38 & 1.45 & 1.46 \\
\hline & 1.39 & 1.50 & 1.54 \\
\hline & 1.44 & 1.50 & 1.55 \\
\hline & 1.45 & 1.50 & 1.56 \\
\hline & 1.48 & 1.50 & 1.59 \\
\hline
\end{tabular}

$\left(\bar{X}_{a, j}, \bar{X}_{b, j}, \bar{X}_{c, j}\right)$ and ranges $\left(R_{a, j}, R_{b, j}, R_{c, j}\right)$ for each sample are given in Table 3 and Table 4.

The regression equations were calculated by using the least squares method with statistical software MINITAB 14.0 where fuzzy arithmetic means were the dependent variable and $t$ (time) the independent variable:

$$
\begin{aligned}
& \bar{X}_{a, j}=\hat{\beta}_{0 a}+\hat{\beta}_{1 a} t_{j}+\varepsilon=1.39+0.00214 t_{j} \\
& \bar{X}_{b, j}=\hat{\beta}_{0 b}+\hat{\beta}_{1 b} t_{j}+\varepsilon=1.43+0.00221 t_{j}
\end{aligned}
$$

$$
\bar{X}_{c, j}=\hat{\beta}_{0 c}+\hat{\beta}_{1 c} t_{j}+\varepsilon=1.47+0.00240 t_{j}
$$

In addition, arithmetic means of fuzzy ranges were obtained by using data from Table 4 , as follows:

$$
\begin{gathered}
\bar{R}_{a}=\frac{\sum_{j=1}^{25} R_{a_{j}}}{25}=0.0104 \\
\bar{R}_{b}=\frac{\sum_{j=1}^{25} R_{b_{j}}}{25}=0.0392 \\
\bar{R}_{c}=\frac{\sum_{j=1}^{25} R_{c_{j}}}{25}=0.154
\end{gathered}
$$

Step 2: Fuzzy $\widetilde{\bar{X}}$-regression and $\widetilde{R}$ control charts were developed by using "Eq.( 29-31)" and "Eq. (81-83) for inner diameters of natural gas valves.

Fuzzy $\widetilde{\bar{X}}$-regression control chart for TFNs case

$$
\begin{aligned}
& U \widetilde{C} L_{\operatorname{Re} g-\bar{X}, j}=\left(\hat{\beta}_{0 a}+\hat{\beta}_{1 a} t_{j}+A_{2} \bar{R}_{a},\right. \\
&\left.\hat{\beta}_{0 b}+\hat{\beta}_{1 b} t_{j}+A_{2} \bar{R}_{b}, \quad \hat{\beta}_{0 c}+\hat{\beta}_{1 c} t_{j}+A_{2} \bar{R}_{c}\right) \\
&=\left(1.39+0.00214 t_{j}+(0.577)(0.0104),\right. \\
& 1.43+0.00221 t_{j}+(0.577)(0.0392), \\
& 1.47+0.00240 t_{j}+(0.577)(0.154) \\
&=\left(1.396+0.00214 t_{j}, 1.452+0.00221 t_{j},\right. \\
&\left.1.558+0.00240 t_{j}\right) \\
& C \widetilde{L}_{\operatorname{Re} g-\bar{X}, j}=\left(\hat{\beta}_{0 a}+\hat{\beta}_{1 a} t_{j},\right.\left.\hat{\beta}_{0 b}+\hat{\beta}_{1 b} t_{j}, \hat{\beta}_{0 c}+\hat{\beta}_{1 c} t_{j}\right) \\
&=\left(1.39+0.00214 t_{j},\right. \\
&\left.1.43+0.00221 t_{j}, \quad 1.47+0.00240 t_{j}\right)
\end{aligned}
$$


Table 3. Fuzzy arithmetic means $\left(\bar{X}_{a, j}, \bar{X}_{b, j}, \bar{X}_{c, j}\right)$ for each sample for the inner diameters of natural gas valves.

\begin{tabular}{|c|c|c|c|}
\hline Time & $\bar{X}_{a, j}$ & $\bar{X}_{b, j}$ & $\bar{X}_{c, j}$ \\
\hline 1 & 1.354 & 1.412 & 1.456 \\
\hline 2 & 1.358 & 1.424 & 1.462 \\
\hline 3 & 1.420 & 1.446 & 1.494 \\
\hline 4 & 1.410 & 1.442 & 1.496 \\
\hline 5 & 1.410 & 1.444 & 1.468 \\
\hline 6 & 1.414 & 1.448 & 1.466 \\
\hline 7 & 1.412 & 1.464 & 1.502 \\
\hline 8 & 1.402 & 1.452 & 1.480 \\
\hline 9 & 1.394 & 1.454 & 1.476 \\
\hline 10 & 1.414 & 1.468 & 1.506 \\
\hline$\vdots$ & $\vdots$ & $\vdots$ & $\vdots$ \\
\hline 21 & 1.430 & 1.474 & 1.524 \\
\hline 22 & 1.426 & 1.476 & 1.520 \\
\hline 23 & 1.430 & 1.476 & 1.522 \\
\hline 24 & 1.438 & 1.48 & 1.518 \\
\hline 25 & 1.438 & 1.482 & 1.514 \\
\hline
\end{tabular}

Table 4. Fuzzy ranges $\left(R_{a, j}, R_{b, j}, R_{c, j}\right)$ for each sample for the inner diameters of natural gas valves.

\begin{tabular}{|c|c|c|c|}
\hline Time & $R_{a, j}$ & $R_{b, j}$ & $R_{c, j}$ \\
\hline 1 & 0.02 & 0.05 & 0.18 \\
\hline 2 & 0.01 & 0.05 & 0.18 \\
\hline 3 & 0.01 & 0.08 & 0.14 \\
\hline 4 & 0.01 & 0.06 & 0.21 \\
\hline 5 & 0.01 & 0.04 & 0.11 \\
\hline 6 & 0.00 & 0.02 & 0.10 \\
\hline 7 & 0.02 & 0.06 & 0.17 \\
\hline 8 & 0.01 & 0.03 & 0.13 \\
\hline 9 & 0.02 & 0.04 & 0.13 \\
\hline 10 & 0.01 & 0.05 & 0.14 \\
\hline$\vdots$ & $\vdots$ & $\vdots$ & $\vdots$ \\
\hline 21 & 0.00 & 0.01 & 0.14 \\
\hline 22 & 0.02 & 0.04 & 0.14 \\
\hline 23 & 0.00 & 0.02 & 0.11 \\
\hline 24 & 0.01 & 0.04 & 0.12 \\
\hline 25 & 0.02 & 0.05 & 0.21 \\
\hline
\end{tabular}

$L \widetilde{C} L_{\operatorname{Re} g-\bar{X}, j}=\left(\hat{\beta}_{0 a}+\hat{\beta}_{1 a} t_{j}-A_{2} \bar{R}_{c}\right.$,

$\left.\hat{\beta}_{0 b}+\hat{\beta}_{1 b} t_{j}-A_{2} \bar{R}_{b}, \hat{\beta}_{0 c}+\hat{\beta}_{1 c} t_{j}-A_{2} \bar{R}_{a}\right)$

$=\left(1.39+0.00214 t_{j}-(0.577)(0.154)\right.$,

$1.43+0.00221 t_{j}-(0.577)(0.0392)$,

$1.47+0.00240 t_{j}-(0.577)(0.0104$

$=\left(1.301+0.00214 t_{j}, 1.407+0.00221 t_{j}\right.$,

$\left.1.463+0.00240 t_{j}\right)$

where, $A_{2}=0.577$ for $n=5, j=1,2, \ldots ., 25$.
Fuzzy $\widetilde{R}$ control chart for TFNs Case

$$
\begin{aligned}
U \widetilde{C} L_{R} & =D_{4}\left(\bar{R}_{a}, \bar{R}_{b}, \bar{R}_{c}\right) \\
& =(2.114)(0.0104,0.0392,0.154) \\
& =(0.0219,0.0828,0.3255) \\
C \widetilde{L}_{R}= & \left(\bar{R}_{a}, \bar{R}_{b}, \bar{R}_{c}\right) \\
= & (0.0104,0.0392,0.154) \\
L \widetilde{C} L_{R} & =D_{3}\left(\bar{R}_{a}, \bar{R}_{b}, \bar{R}_{c}\right) \\
& =(0)(0.0104,0.0392,0.154) \\
& =(0,0,0)
\end{aligned}
$$

where $D_{4}=2.114$ and $D_{3}=0$ for $\mathrm{n}=5$.

Step 3: The $\alpha$-cut fuzzy $\tilde{\bar{X}}$-regression and $\widetilde{R}$ control charts were formulated by integrating an $\alpha$ cut, where $\alpha=0.75$ was selected as a result of the manufacturing process, which has a trend that is more critical than the classic ones. The $\alpha$-cut for the regression equation of fuzzy means and the $\alpha$-cut fuzzy means of ranges can be calculated as follows:

$$
\begin{aligned}
& \tilde{\bar{X}}_{a, j}^{0.75}=(1-\alpha)\left(\hat{\beta}_{0 a}-\hat{\beta}_{1 a} t_{j}\right)+\alpha\left(\hat{\beta}_{0 b}+\hat{\beta}_{1 b} t_{j}\right) \\
& =(1-0.75)\left(1.39-0.00214 t_{j}\right)+0.75\left(1.43+0.00221 t_{j}\right) \\
& =1.42+0.0011225 t_{j}
\end{aligned}
$$

$$
\begin{aligned}
& \tilde{\bar{X}}_{c, j}^{0.75}=(1-\alpha)\left(\hat{\beta}_{0 c}-\hat{\beta}_{1 c} t_{j}\right)+\alpha\left(\hat{\beta}_{0 b}+\hat{\beta}_{1 b} t_{j}\right) \\
& =(1-0.75)\left(1.47-0,00240 t_{j}\right)+0.75\left(1.43+0.00221 t_{j}\right) \\
& =1.44+0.0010575 t_{j}
\end{aligned}
$$

$$
\bar{R}_{a}^{0.75}=\bar{R}_{a}+\alpha\left(\bar{R}_{b}-\bar{R}_{a}\right)
$$$$
=0.0104+0.75(0.0392-0.0104)=0.032
$$

$$
\begin{aligned}
& \bar{R}_{c}^{0.75}=\bar{R}_{c}-\alpha\left(\bar{R}_{c}-\bar{R}_{b}\right) \\
& =0.154-0.75(0.154-0.0392)=0.068
\end{aligned}
$$

The $\alpha$-cut fuzzy $\widetilde{\bar{X}}$-regression and fuzzy $\widetilde{R}$ control charts were expressed by using $\tilde{\bar{X}}_{a, j}^{0.75}, \widetilde{\bar{X}}_{c, j}^{0.75}, \bar{R}_{a}^{0.75}$ and $\bar{R}_{c}^{0.75}$ and Eq. 36-38.

The $\alpha$-cut fuzzy $\tilde{\bar{X}}$-regression control chart for TFNs: 


$$
\begin{aligned}
& U \widetilde{C} L_{\operatorname{Re} g-\bar{X}, j}^{0.75}=\left(\bar{X}_{a, j}^{\alpha}+A_{2} \bar{R}_{a}^{\alpha},\right.\left.\bar{X}_{b, j}+A_{2} \bar{R}_{b}, \bar{X}_{c, j}^{\alpha}+A_{2} \bar{R}_{c}^{\alpha}\right) \\
&=\left(1.42+0.0011225 t_{j}+(0.577)(0.032),\right. \\
& 1.43+0.00221 t_{j}+(0.577)(0.0392), \\
&\left.1.44+0.0010575 t_{j}+(0.577)(0.068)\right) \\
&=\left(1.438+0.0011225 t_{j}, 1.452+0.00221 t_{j},\right. \\
&\left.1.479+0.0010575 t_{j}\right) \\
& \widetilde{C} L_{\operatorname{Re} g-\bar{X}, j}^{0.75}=\left(\bar{X}_{a, j}^{\alpha}, \bar{X}_{b, j}, \bar{X}_{c, j}^{\alpha}\right) \\
&=\left(1.42+0.0011225 t_{j}, 1.43+0.00221 t_{j},\right. \\
&\left.1.44+0.0010575 t_{j}\right) \\
& L \widetilde{C} L_{\operatorname{Re} g-\bar{X}, j}^{0.75}=\left(\bar{X}_{a, j}^{\alpha}-A_{2} \bar{R}_{c}^{\alpha},\right. \\
&\left.\bar{X}_{b, j}-A_{2} \bar{R}_{b}, \bar{X}_{c, j}^{\alpha}-A_{2} \bar{R}_{a}^{\alpha}\right) \\
&=\left(1.42+0.0011225 t_{j}-(0.577)(0.068),\right. \\
& 1.43+0.00221 t_{j}-(0.577)(0.0392), \\
&\left.1.44+0.0010575 t_{j}-(0.577)(0.032)\right) \\
&= 1.380+0.0011225 t_{j}, 1.407+0.00221 t_{j}, \\
& 1.421+0.0010575 t_{j}
\end{aligned}
$$

The $\alpha$-cut fuzzy $\widetilde{R}$ control chart for TFNs

$$
\begin{aligned}
& U \widetilde{C} L_{R}^{0.75}=D_{4}\left(\bar{R}_{a}^{\alpha}, \bar{R}_{b}, \bar{R}_{c}^{\alpha}\right) \\
& =(2.114)(0.032,0.0392,0.068) \\
& =(0.0676,0.0828,0.1437) \\
& C \widetilde{L}_{R}^{0.75}=\left(\bar{R}_{a}^{\alpha}, \bar{R}_{b}, \bar{R}_{c}^{\alpha}\right) \\
& =(0.032,0.0392,0.068) \\
& L \widetilde{C} R_{R}^{0.75}=D_{3}\left(\bar{R}_{a}^{\alpha}, \bar{R}_{b}, \bar{R}_{c}^{\alpha}\right) \\
& =(0)(0.032,0.0392,0.068) \\
& =(0,0,0)
\end{aligned}
$$

Step 4: The $\alpha$-level fuzzy midrange for the $\alpha$-cut fuzzy $\widetilde{\bar{X}}$-regression and $\widetilde{R}$ control charts were derived for inner diameters of natural gas valves:

$$
\begin{aligned}
& U C L_{m r-\mathrm{Reg}-\bar{X}, j}^{0.75}=\frac{\left(\bar{X}_{a, j}^{\alpha}+\bar{X}_{c, j}^{\alpha}\right)}{2}+A_{2}\left(\frac{\bar{R}_{a}^{\alpha}+\bar{R}_{c}^{\alpha}}{2}\right) \\
& =\frac{\left(1.42+0.0011225 t_{j}+1.44+0.0010575 t_{j}\right)}{2} \\
& +(0.577)\left(\frac{0.032+0.068}{2}\right) \\
& =1.46+0.00109 t_{j}
\end{aligned}
$$

$$
\begin{aligned}
& C L_{m r-\operatorname{Re} g-\bar{X}, j}^{0.75}=\frac{\left(\bar{X}_{a, j}^{\alpha}+\bar{X}_{c, j}^{\alpha}\right)}{2} \\
& =\frac{\left(1.42+0.0011225 t_{j}+1.44+0.0010575 t_{j}\right)}{2} \\
& =1.43+0.00109 t_{j} \\
& L C L_{m r-\operatorname{Re} g-\bar{X}, j}^{0.75}=\frac{\left(\bar{X}_{a, j}^{\alpha}+\bar{X}_{c, j}^{\alpha}\right)}{2}-A_{2}\left(\frac{\bar{R}_{a}^{\alpha}+\bar{R}_{c}^{\alpha}}{2}\right) \\
& =\frac{\left(1.42+0.0011225 t_{j}+1.44+0.0010575 t_{j}\right)}{2} \\
& -(0.577)\left(\frac{0.032+0.068}{2}\right) \\
& =1.40+0.00109 t_{j}
\end{aligned}
$$

where $A_{2}=0.577$ for $n=5$.

The $\alpha$-level fuzzy midrange for the $\alpha$-cut fuzzy $\widetilde{R}$ was derived for inner diameters of natural gas valves:

$$
\begin{aligned}
& U C L_{m r-R}^{0.75}=D_{4} f_{m r-R}^{\alpha}(C \tilde{L}) \\
& =(2.114)\left(\frac{0.032+0.068}{2}\right) \\
& =0.1057 \\
& C L_{m r-R}^{0.75}=\frac{\bar{R}_{a}^{\alpha}+\bar{R}_{c}^{\alpha}}{2} \\
& =\left(\frac{0.032+0.068}{2}\right) \\
& =0.05 \\
& L C L_{m r-R}^{0.75}=D_{3} f_{m r-R}^{\alpha}(C \widetilde{L}) \\
& =(0)\left(\frac{0.032+0.068}{2}\right) \\
& =0
\end{aligned}
$$

where $D_{4}=2.114$ and $D_{3}=0$ for $n=5$.

Step 5: The process condition was set to evaluate the process with an $\alpha$-cut based on the $\alpha$-level fuzzy midrange transformation technique for the inner diameter of the natural gas valves. $S_{m r-\operatorname{Re} g-\bar{X}, j}^{\alpha}$ and $S_{m r-R, j}^{\alpha}$ were calculated for 25 samples by using "Eq. (43)", "Eq.(90)", respectively, and are given in Table 5.and Table6.

As shown in Table 5 the process is "out of control" for only the last sample. Before that, the process was "under control" with respect to $S_{m r-\operatorname{Re} g-\bar{X}, j}^{\alpha}$. When examining the deviation of the process, all samples showed that the process is "under control" 
with respect to $S_{m r-R, j}^{\alpha}$ in Table 6. It could be said that the process is "out of control" because of the $\alpha$ level fuzzy midrange for the $\alpha$-cut fuzzy $\widetilde{\bar{X}}$ regression control chart. The tool should be changed before operating the $25^{\text {th }}$ sample. In this way, the end of the process should be under control. The life of insert is sufficient up to the $24^{\text {th }}$ sample. Thus, it can be said that insert wearing is not a problem for inner diameters the of natural gas valves.

Table 5. Control limits using the $\alpha$-level fuzzy midrange for the $\alpha$-cut fuzzy $\widetilde{\bar{X}}$-regression control chart based on ranges.

\begin{tabular}{|c|c|c|c|}
\hline $\mathrm{t}$ & $S_{m r-\bar{X}, j}^{\alpha}$ & $\begin{array}{l}1.40+0.00109 t_{j} \\
\leq S_{m r-\operatorname{Re} g-\bar{X}, j}^{\alpha} \leq \\
1.46+0.00109 t_{j}\end{array}$ & $\begin{array}{l}\text { Process } \\
\text { Condition } \\
\end{array}$ \\
\hline 1 & 1.410 & $1.401 \leq S_{m r-\operatorname{Re} g-\bar{X}, j}^{\alpha} \leq 1.461$ & under control \\
\hline 2 & 1.421 & $1.402 \leq S_{m r-\operatorname{Re} g-\bar{X}, j}^{\alpha} \leq 1.462$ & under control \\
\hline 3 & 1.449 & $1.403 \leq S_{m r-\operatorname{Re} g-\bar{X}, j}^{\alpha} \leq 1.463$ & under control \\
\hline 4 & 1.445 & $1.404 \leq S_{m r-\operatorname{Re} g-\bar{X}, j}^{\alpha} \leq 1.464$ & under control \\
\hline 5 & 1.443 & $1.405 \leq S_{m r-\operatorname{Re} g-\bar{X}, j}^{\alpha} \leq 1.465$ & under control \\
\hline 6 & 1.446 & $1.407 \leq S_{m r-\operatorname{Re} g-\bar{X}, j}^{\alpha} \leq 1.467$ & under control \\
\hline 7 & 1.462 & $1.408 \leq S_{m r-\operatorname{Re} g-\bar{X}, j}^{\alpha} \leq 1.468$ & under control \\
\hline 8 & 1.449 & $1.409 \leq S_{m r-\operatorname{Re} g-\bar{X}, j}^{\alpha} \leq 1.469$ & under control \\
\hline 9 & 1.449 & $1.410 \leq S_{m r-\operatorname{Re} g-\bar{X}, j}^{\alpha} \leq 1.470$ & under control \\
\hline 10 & 1.466 & $1.411 \leq S_{m r-\operatorname{Re} g-\bar{X}, j}^{\alpha} \leq 1.471$ & under control \\
\hline 11 & 1.466 & $1.412 \leq S_{m r-\operatorname{Re} g-\bar{X}, j}^{\alpha} \leq 1.472$ & under control \\
\hline 12 & 1.456 & $1.413 \leq S_{m r-\operatorname{Re} g-\bar{X}, j}^{\alpha} \leq 1.473$ & under control \\
\hline 13 & 1.450 & $1.414 \leq S_{m r-\operatorname{Re} g-\bar{X}, j}^{\alpha} \leq 1.474$ & under control \\
\hline 14 & 1.471 & $1.415 \leq S_{m r-\operatorname{Re} g-\bar{X}, j}^{\alpha} \leq 1.475$ & under control \\
\hline 15 & 1.466 & $1.416 \leq S_{m r-\operatorname{Re} g-\bar{X}, j}^{\alpha} \leq 1.476$ & under control \\
\hline 16 & 1.461 & $1.417 \leq S_{m r-\operatorname{Re} g-\bar{X}, j}^{\alpha} \leq 1.477$ & under control \\
\hline 17 & 1.465 & $1.419 \leq S_{m r-\operatorname{Re} g-\bar{X}, j}^{\alpha} \leq 1.479$ & under control \\
\hline 18 & 1.472 & $1.420 \leq S_{m r-\operatorname{Re} g-\bar{X}, j}^{\alpha} \leq 1.480$ & under control \\
\hline 19 & 1.474 & $1.421 \leq S_{m r-\operatorname{Re} g-\bar{X}, j}^{\alpha} \leq 1.481$ & under control \\
\hline 20 & 1.475 & $1.422 \leq S_{m r-\operatorname{Re} g-\bar{X}, j}^{\alpha} \leq 1.482$ & under control \\
\hline 21 & 1.475 & $1.423 \leq S_{m r-\operatorname{Re} g-\bar{X}, j}^{\alpha} \leq 1.483$ & under control \\
\hline 22 & 1.476 & $1.424 \leq S_{m r-\operatorname{Re} g-\bar{X}, j}^{\alpha} \leq 1.484$ & under control \\
\hline 23 & 1.480 & $1.425 \leq S_{m r-\operatorname{Re} g-\bar{X}, j}^{\alpha} \leq 1.485$ & under control \\
\hline 24 & 1.481 & $1.426 \leq S_{m r-\operatorname{Re} g-\bar{X}, j}^{\alpha} \leq 1.486$ & under control \\
\hline 25 & 1.489 & $1.427 \leq S_{m r-\operatorname{Re} g-\bar{X}, j}^{\alpha} \leq 1.487$ & Out of control \\
\hline
\end{tabular}


Table 6. Control limits using the $\alpha$-level fuzzy midrange for an $\alpha$-cut fuzzy $\widetilde{R}$ control chart.

\begin{tabular}{|c|c|c|c|}
\hline $\mathrm{t}$ & $S_{m r-R, j}^{\alpha}$ & $0 \leq S_{m r-R, j}^{\alpha} \leq 0.1057$ & Process Condition \\
\hline 1 & 0.06 & $0 \leq S_{m r-R, j}^{\alpha} \leq 0.1057$ & under control \\
\hline 2 & 0.06 & $0 \leq S_{m r-R, j}^{\alpha} \leq 01057$ & under control \\
\hline 3 & 0.08 & $0 \leq S_{m r-R, j}^{\alpha} \leq 0.1057$ & under control \\
\hline 4 & 0.07 & $0 \leq S_{m r-R, j}^{\alpha} \leq 0.1057$ & under control \\
\hline 5 & 0.05 & $0 \leq S_{m r-R, j}^{\alpha} \leq 0.1057$ & under control \\
\hline 6 & 0.03 & $0 \leq S_{m r-R, j}^{\alpha} \leq 0.1057$ & under control \\
\hline 7 & 0.07 & $0 \leq S_{m r-R, j}^{\alpha} \leq 0.1057$ & under control \\
\hline 8 & 0.04 & $0 \leq S_{m r-R, j}^{\alpha} \leq 0.1057$ & under control \\
\hline 9 & 0.05 & $0 \leq S_{m r-R, j}^{\alpha} \leq 0.1057$ & under control \\
\hline 10 & 0.06 & $0 \leq S_{m r-R, j}^{\alpha} \leq 0.1057$ & under control \\
\hline 11 & 0.06 & $0 \leq S_{m r-R, j}^{\alpha} \leq 0.1057$ & under control \\
\hline 12 & 0.03 & $0 \leq S_{m r-R, j}^{\alpha} \leq 0.1057$ & under control \\
\hline 13 & 0.08 & $0 \leq S_{m r-R, j}^{\alpha} \leq 0.1057$ & under control \\
\hline 14 & 0.06 & $0 \leq S_{m r-R, j}^{\alpha} \leq 0.1057$ & under control \\
\hline 15 & 0.06 & $0 \leq S_{m r-R, j}^{\alpha} \leq 0.1057$ & under control \\
\hline 16 & 0.04 & $0 \leq S_{m r-R, j}^{\alpha} \leq 0.1057$ & under control \\
\hline 17 & 0.03 & $0 \leq S_{m r-R, j}^{\alpha} \leq 0.1057$ & under control \\
\hline 18 & 0.04 & $0 \leq S_{m r-R, j}^{\alpha} \leq 0.1057$ & under control \\
\hline 19 & 0.04 & $0 \leq S_{m r-R, j}^{\alpha} \leq 0.1057$ & under control \\
\hline 20 & 0.03 & $0 \leq S_{m r-R, j}^{\alpha} \leq 0.1057$ & under control \\
\hline 21 & 0.03 & $0 \leq S_{m r-R, j}^{\alpha} \leq 0.1057$ & under control \\
\hline 22 & 0.05 & $0 \leq S_{m r-R, j}^{\alpha} \leq 0.1057$ & under control \\
\hline 23 & 0.03 & $0 \leq S_{m r-R, j}^{\alpha} \leq 0.1057$ & under control \\
\hline 24 & 0.05 & $0 \leq S_{m r-R, j}^{\alpha} \leq 0.1057$ & under control \\
\hline 25 & 0.07 & $0 \leq S_{m r-R, j}^{\alpha} \leq 0.1057$ & under control \\
\hline
\end{tabular}




\section{Conclusion}

A regression control chart is generally used for the tool wearing problem. When manufacturing processes and/or measurement systems have some uncertainty and vagueness due to operators or gauges, the fuzzy $\widetilde{\bar{X}}$-regression control chart is more suitable than a traditional regression control chart for analyzing the tool wearing problem. In addition the deviation can be monitored with a fuzzy $\widetilde{R}$ control chart.

The aim of this study is to present the theoretical structure of the " $\alpha$-level fuzzy midrange for the $\alpha$-cut fuzzy $\widetilde{\bar{X}}$-regression control chart". The proposed fuzzy $\widetilde{\bar{X}}$-regression control chart is illustrated with a numerical example of threading inner diameters of a natural gas valve. In addition, the fuzzy $\widetilde{R}$ control chart is implemented to monitor the variation of inner diameters of the natural gas valve.

Many natural gas valves can be threaded with one insert. Thus, tool wearing problems occur in the manufacturing process. The threading process is "out of control" according to fuzzy $\widetilde{\bar{X}}$-regression control chart. It should be controlled at the end of the manufacturing process. The deviation is "under control" according to the fuzzy $\widetilde{R}$ control chart.

In fuzzy control charts, an $\alpha$-cut represents the ability to determine the tightness of the collection sample process. The fuzzy midrange transformation technique can transform the fuzzy samples to a crisp number. Other transformation techniques and membership functions can be applied to obtain an $\alpha$-cut fuzzy $\widetilde{\bar{X}}$-regression control chart for further research.

\section{References}

1. D.C. Montgomery.and C. Mastrangelo. Some statistical process control methods for autocorrelated data. Journal of Quality Technology. 23(3) (1991) 179-193.

2. D.C. Montgomery. Introduction to Statistical Quality Control. (John Wiley \& Sons. Inc. USA. 1991).

3. A.J. Duncan. Quality Control and Industrial Statistics. 4 th edn. (Irwin. Homewood. III. 1974).
4. J. Manuele. Control chart for determining tool wear. Industrial Quality Control. 1 (1945).

5. J. Mandel. The Regression Control Chart. Journal Of Quality Technology. 1 (1969).

6. C.P.Quesenberry. An SPC Approach to compensating a tool-wear process. Journal of Quality Technology. 20 (1988).

7. L.A. Zadeh. Fuzzy Sets. Information and Control. 8 (1965) 338-353.

8. J.H. Wang and T. Raz. On the construction of control charts using linguistic variables. Intelligent Journal of Production Research. 28 (1990) 477-487.

9. T. Raz and J.H. Wang. Probabilistic and memberships approaches in the construction of control chart for linguistic data. Production Planning and Control.1 (1990) 147-157.

10. A.Kanagawa. F. Tamaki and H. Ohta. Control charts for process average and variability based on linguistic data. Intelligent Journal of Production Research. 3(4) (1993) 913-922.

11. H. Rowlands and L.R. Wang. An approach of fuzzy logic evaluation and control in SPC. Quality Reliability Engineering Intelligent. 16 (2000) 91-98.

12. S.M. El-Shal and A.S. Morris. A fuzzy rule-based algorithm to improve the performance of statistical process control in quality systems. Journal of Intelligent Fuzzy Systems. 9 (2000) 207-223.

13. H.M.Hsu and Y.K. Chen. A fuzzy reasoning based diagnosis system for $\bar{X}$ control charts. Journal of Intelligent Manufacturing. 12 (2001) 57-64.

14. M.Gülbay. C. Kahraman and D. Ruan. $\alpha$-cut fuzzy control chart for linguistic data. International. Journal of Intelligent Systems. 19 (2004) 1173-1196.

15. C.B. Cheng. Fuzzy process control: construction of control charts with fuzzy numbers. Fuzzy sets and Systems. 154 (2005) 287-303.

16. M.Gülbay and C. Kahraman. Development of fuzzy process control charts and fuzzy unnatural pattern analyses. Computational Statistics and Data Analysis. 51 (2006a) 434-451.

17. M.Gülbay and C. Kahraman. An alternative approach to fuzzy control chart: Direct fuzzy approach. Information Sciences. 77(6) (2006b) 1463-1480. 
18. A.Faraz and M.B. Moghadam. Fuzzy control chart a beter alternative for Shewhart Average Chart. Quality \& Quantity. 41 (2007) 375-385.

19. K.L. Hsieh. L.I. Tong and M.C. Wang. The application of control chart for defects and defect clustering in IC manufacturing based on fuzzy theory. Expert Systems with Applications. 32 (2007) 765-776.

20. M.H.F. Zarandi. A. Alaeddini and I.B. Turksen. A hybrid fuzzy adaptive sampling - Run rules for Shewhart control charts. Information Sciences. 178 (2008) 1152-1170.

21. O. Engin. A. Çelik and İ. Kaya. A fuzzy approach to define sample size for attributes control chart in multistage processes: An application in engine valve manufacturing process. Applied Soft Computing. 8 (2008) 1654-1663.

22. N.Erginel. Fuzzy individual and moving range control charts with $\alpha$-cuts. Journal of Intelligent $\&$ Fuzzy Systems. 19 (2008) 373-383.

23. A. Alaeddini. M. Ghazanfari and M.A. Nayeri. A hybrid fuzzy-statistical clustering approach for estimating the time of changes in fixed and variable sampling control charts. Information Sciences. 179 (2009) 1769-1784.

24. S. Şentürk and N. Erginel. Development of fuzzy $\stackrel{\widetilde{X}}{-} \widetilde{R}$ and $\stackrel{\widetilde{X}}{-} \widetilde{S}$ control charts using $\alpha$-cuts. Information Sciences. 179 (2009) 1542-1551.

25. S. Şentürk. Fuzzy regression control chart. in Proc. of the 8th International FLINS (Fuzzy Logic and Intelligent Technologies in Nuclear Science) Conference. (Madrid. Spain. 2008). pp. 963-968.

26. W.J. Kolarik. Creating Quality-Concepts. Systems. Strategies and Tools. (McGraw Hill. 1995).

27. N. Melouah. H. Kherfane and B. Bensaker. fuzzy sets- based nonlinear modeling of induction machines: an alpha-cut approach. International Journal of Electrical and Power Engineering. 14 (2007) 438-442.

28. H.J. Zimmermann.. Fuzzy set theory- and its applications. Kluwer Academic Publishers. Boston. Dordrecht. London. 4th edition. 2001. 